\title{
On Detection of Black Hole Quasi-Normal Ringdowns: Detection Efficiency and Waveform Parameter Determination in Matched Filtering
}

\author{
Yoshiki Tsunesada ${ }^{1}$, Nobuyuki Kanda ${ }^{2}$, Hiroyuki Nakano ${ }^{2}$, Daisuke Tatsumi ${ }^{1}$, \\ Masaki Ando $^{3}$, Misao Sasaki ${ }^{4}$, Hideyuki Tagoshi ${ }^{5}$, and Hirotaka Takahashi ${ }^{5,6}$ \\ ${ }^{1}$ National Astronomical Observatory of Japan, Tokyo 181-8588, Japan \\ ${ }^{2}$ Department of Mathematics And Physics, Graduate School of Science, Osaka City University, Osaka 558-8585, Japan \\ ${ }^{3}$ Department of Physics, University of Tokyo, Tokyo 113-0033, Japan \\ ${ }^{4}$ Yukawa Institute for Theoretical Physics, Kyoto University, Kyoto 606-8502, Japan \\ ${ }^{5}$ Department of Earth and Space Science, Graduate School of Science, Osaka University, Osaka 560-0043, Japan and \\ ${ }^{6}$ Graduate School of Science and Technology, Niigata University, Niigata 950-2181, Japan
}

\begin{abstract}
Gravitational radiation from a slightly distorted black hole with ringdown waveform is well understood in general relativity. It provides a probe for direct observation of black holes and determination of their physical parameters, masses and angular momenta (Kerr parameters). For ringdown searches using data of gravitational wave detectors, matched filtering technique is useful. In this paper, we describe studies on problems in matched filtering analysis in realistic gravitational wave searches using observational data. Above all, we focus on template constructions, matches or signal-to-noise ratios (SNRs), detection probabilities for Galactic events, and accuracies in evaluation of waveform parameters or black hole hairs. In template design for matched filtering, search parameter ranges and template separations are determined by requirements from acceptable maximum loss of SNRs, detection efficiencies, and computational costs. In realistic searches using observational data, however, effects of non-stationary noises cause decreases of SNRs, and increases of errors in waveform parameter determinations. These problems will potentially arise in any matched filtering searches for any kind of waveforms. To investigate them, we have performed matched filtering analysis for artificial ringdown signals which are generated with Monte-Carlo technique and injected into the TAMA300 observational data. We employed an efficient method to construct a bank of ringdown filters recently proposed by Nakano et al., and use a template bank generated from a criterion such that losses of SNRs of any signals do not exceed 2\%. We found that this criterion is fulfilled in ringdown searches using TAMA300 data, by examining distribution of SNRs of simulated signals. It is also shown that with TAMA300 sensitivity, the detection probability for Galactic ringdown events is about $50 \%$ for black holes of masses greater than $20 M_{\odot}$ with $\mathrm{SNR}>10$. The accuracies in waveform parameter estimations are found to be consistent with the template spacings, and resolutions for black hole masses and the Kerr parameters are evaluated as a few $\%$ and $\sim 40 \%$, respectively. They can be improved up to $<0.9 \%$ and $<24 \%$ for events of SNR $\geq 10$ by using fine-meshed template bank in the hierarchical search strategy.
\end{abstract}

PACS numbers: 95.85.Sz, 04.80Nn, 07.05.Kf, 97.60.Lf

\section{INTRODUCTION}

With recent progress in the development of gravitational wave detectors as resonant bars [1] and laser interferometers [2, 3, 4], the detectors' sensitivities and operational stabilities have significantly been improved. Several groups have performed long-term observations which provided data of good quality suitable for various kind of astrophysical gravitational wave searches. These are for "chirp" signals from inspiralling compact neutron star binaries, burst-like signals from stellar core collapses in supernovae, stochastic gravitational wave background, and continuous waves from pulsars. Thus well organized studies on data analysis techniques are required for gravitational wave detection and gravitational wave astronomy in near future. For some classes of gravitational wave sources, data analysis techniques have already been investigated extensively, in particular as for inspiral searches. The matched filtering technique is considered as one of the best method for this, since the gravitational waveform is theoretically predicted in good ac- curacy. Although it is rather difficult to exploit an optimal method for gravitational wave bursts with undefined waveforms, several methods and filters have been proposed (e.g. [5, 6] ).

Gravitational radiation from a perturbed black hole, so called ringdown, is one of the classes of known-waveform gravitational wave sources. The black hole perturbation in the late stage of a black hole formation, initiated by a coalescence of a binary system for example, can be described by quasi-normal modes (QNMs). Therefore gravitational radiation is expected as of damped sinusoidal waveform. This is represented in time domain by

$$
h(t) \propto \exp \left(-\frac{\pi f_{c}}{Q}\left(t-t_{0}\right)\right) \sin \left(2 \pi f_{c}\left(t-t_{0}\right)+\phi_{0}\right)
$$

where $f_{c}, Q, \phi_{0}, t_{0}$ are the central frequency, the quality factor of the ringing, the initial phase, and the arrival time, respectively. The QNMs were studied extensively by using Regge-Wheeler-Zerilli or Teukolsky formalism in 1970's and 1980's (e.g. 7, [8, 9, 10]), and several methods have been proposed for numerical computation of 
them (11, 12, 13, 14], and 15] for review and references therein). Leaver developed a numerically stable method for calculation of QNMs with good accuracies [16], and his results have been verified by many authors with independent schemes. For the least damped mode of the QNM with $l=m=2$ spin-2 spheroidal harmonics ${ }_{-2} S_{22}$, Echeverria have given analytic expressions for $f_{c}$ and $Q$ as functions of the black hole mass $M$ and the non-dimensional angular momentum (the Kerr parameter) $a=[0,1)$, as $[17]$

$$
\begin{aligned}
f_{c}[\mathrm{kHz}] & \simeq 32\left(\frac{M}{M_{\odot}}\right)^{-1} \times\left[1-\alpha(1-a)^{\beta}\right] \\
Q & \simeq 2(1-a)^{\gamma}
\end{aligned}
$$

where $\beta=3 / 10, \gamma=-9 / 20$ and $\alpha=63 / 100$. These expressions give ringdown parameters $\left(f_{c}, Q\right)$ with accuracies of $\sim 5 \%$. For black holes with masses of $10 \sim 200 M_{\odot}$, the ringdown frequencies fall in the observational band $100 \mathrm{~Hz} \sim \mathrm{kHz}$ of ground-based laser interferometric detectors. Matched filtering technique is useful to find ringdown signals in outputs of gravitational wave detectors since their waveforms are modeled in a simple form as equation (1) in terms of the parameters $\left(f_{c}, Q\right)$ which can be easily converted into the black hole parameters $(M, a)$.

The black hole ringdowns are very interesting in gravitational wave astronomy from some aspects. First, ringdown detection will be a direct confirmation of the existence of astrophysical black holes. Second, it gives an another "mass window" for observation of astrophysical objects by detecting gravitational waves. For example, for black hole binaries with several tens of solar-masses, frequencies of gravitational radiation in the inspiralling phase are out of the observational band of contemporary laser interferometers, while they can be detected via ringdown waves. In spite of its significance, there are fewer publications on ringdown searches compared to those for inspiral or burst searches. Creighton reported the results of a single filter search for a black hole with a particular mass and spin using data of the Caltech 40-m interferometer 18]. Arnaud et al. discussed a general template construction method for matched filtering searches and its application to the ringdown case [19]. Recently Nakano et al. proposed an efficient tiling method for ringdown filters [20, 21].

The aim of this paper is to discuss problems in matched filtering analysis in realistic search using observational data. In matched filtering with a template bank, the template separation is taken so that losses of signal-tonoise-ratios (SNRs) of any signals do not exceed a preselected value (minimal match), $M M$. The choice of $M M$ determines detection efficiencies and waveform parameter resolutions. Some template construction methods for a given $M M$ have already been proposed as 19], 20, 21]. In a realistic search, however, non-stationary noises in detector outputs would reduce SNRs, so it is not clear whether the criterion $\geq M M$ is still fulfilled.
Furthermore, the template which gives the maximum of the matches is not necessarily the nearest to the signal, thus the noise effects would also appear in waveform parameter resolutions. To investigate these problems, we have carried out matched filtering searches for artificial ringdown signals embedded in TAMA300 data with Monte-Carlo technique. The ringdown signals with randomly selected waveform parameters are injected into TAMA300 data by software, and filtered with the template bank. We employ the template spacing method recently proposed by Nakano et al. [20, 21], which provides an efficient tiling scheme to cover the parameter space with a fewer templates keeping the criterion $\geq M M$. For gravitational wave sources, we consider black holes distributed in the Galaxy, since magnitudes of ringdown waves from distances of Galactic scales $(\sim 10 \mathrm{kpc})$ are expected enough large to be detected with contemporary interferometors as TAMA300 (see FIG. (1). From the simulation results, we evaluated SNRs and their losses, detection efficiencies with given SNR thresholds, and the waveform parameter resolutions. We also discuss possibility of black hole "spectroscopy" with gravitational wave observations by using very fine-meshed template bank and hierarchical search technique. The code used here is the same which will be used in full-scale event searches using TAMA300 data. The information obtained in this work are important for astrophysical interpretation of search results and to set an observational limit on the ringdown event rate.

The plan of the remaining part of the paper is as follows. In Sec. II, we briefly describe the matched filtering technique and the template construction method employed here. The details of our simulation studies are given in Sec. III, and the results are shown in Sec. IV. Section V is devoted to discussion. Finally, in Sec. VI, we summarize and conclude our work.

\section{MATCHED FILTERING}

\section{A. Template spacings}

The matched filtering method is to compute a crosscorrelation (match) between detector outputs and each of reference waveforms as a "template" characterized with waveform parameter(s), $X^{\mu}=\left(X^{1}, X^{2}\right)=\left(f_{c}, Q\right)$ in ringdown case, to find signals possibly embedded in the detector outputs. The match between a signal $s(t)$ and a template $h(t)$ with the $k$-th waveform parameter $X_{k}^{\mu}$ is expressed in Fourier domain as

$$
\rho\left(X_{k}^{\mu}\right)=\left\langle s \mid h\left(X_{k}^{\mu}\right)\right\rangle \propto \int d f \frac{\tilde{s}(f) \tilde{h}^{*}\left(f ; X_{k}^{\mu}\right)}{S_{n}(|f|)}
$$

where $\tilde{s}(f)$ and $\tilde{h}(f)$ express the Fourier transforms of functions of time $s(t)$ and $h(t)$, the asterisk $(*)$ denotes the complex conjugation, and $S_{n}(|f|)$ is the power spectrum of the detector noise. In a practical search, the 
signal $s$ in equation (4) is replaced by the sum with detector noise $n$, namely $o=n+s$. The template bank is produced so that for any signals there exists at least one template which gives the match greater than the minimal match, as $\left\langle\hat{h}\left(X^{\mu}\right) \mid \hat{h}\left(X_{k}^{\mu}\right)\right\rangle \geq M M$ for ${ }^{\exists} X_{k}^{\mu}$, where $\hat{h}$ is normalized as $\langle\hat{h} \mid \hat{h}\rangle=1$. The choice of $M M$ is from statistical or astrophysical requirements (a larger $M M$ reduces matches in average and hence detection efficiencies), and computational tolerance (a smaller $M M$ results in an increase of total number of templates). Thus the key of template construction is how cover the parameter space with a fewer templates for a given $M M$.

A mismatch of two waveforms, defined as 1 - (match), can be understood as a distance between them. Thus to obtain appropriate template spacings and "tile" the parameter space, it is required to calculate the metric of the space 22]. As seen from equation (4), the metric of the parameter space is determined by the waveform $h$ and the power spectrum of detector noise $S_{n}(|f|)$. In our previous papers [20, 21], we discussed a tiling method in the frequency domain, assuming the detector noise is white, i.e., $S_{n}(|f|)=$ const. in equation (4). In this case, the metric and the template tiling can be formulated analytically, hence it is easy to program the template spacing scheme in a search code.

In [20, 21], we also discussed the validity of a use of this template bank in the case of a detector's colored noise power spectrum. As a model of realistic noise power spectrum, we used a fitting curve (without lines, structures, fluctuations) of the noise power spectrum of TAMA300 at Data Taking 8 in 2003. We prepare a template bank using the above spacing. Signals are generated so that their amplitudes are normalized to unity. Then, we perform the matched filtering and evaluate the maximum of the match for each signal. It is found that, in most cases (99.12\% in our calculation), the losses in SNR are not greater than $2 \%$ which is expected from the pre-assigned minimal match for the white noise case. This shows that the template spacing can be used even in the case of colored noise power spectrum. The mean of the match is 0.993. However, in the real data analysis, we must treat non-stationary noise fluctuations in detector's output. In the following, we discuss the simulated ringdown signals injected into TAMA300 data.

\section{B. Matched Filter Code}

The matched filtering code used here has developed at National Astronomical Observatory of Japan (NAOJ) and at Osaka City University (OCU). The TAMA300 data are split into data chunks of about $52 \mathrm{sec}$, and the matched filtering code are applied for each of them. We use the moving-average method to obtain the noise power spectrum of detector outputs $S_{n}(|f|)$ as a frequency weighting function in equation (4) in the calculation of cross-correlations [23]. We define the parame-

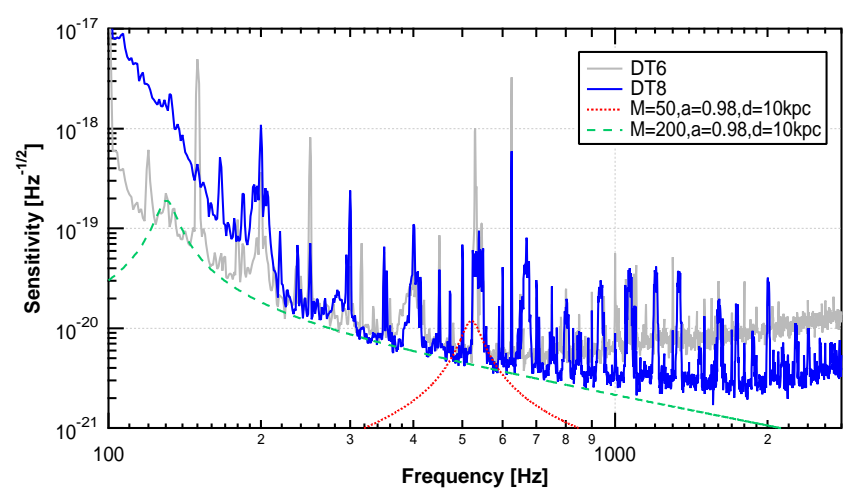

FIG. 1: TAMA300 sensitivity curves for DT6 (thin) and DT8 (thick). Ringdown spectra are also shown for black holes of $M=50 \mathrm{M}_{\odot}\left(\right.$ dotted) and $M=200 \mathrm{M}_{\odot}$ (dashed) with a fixed Kerr parameter $a=0.98$ and a distance $d=10 \mathrm{kpc}$.

ter ranges for $f_{c}$ and $Q$ as $1.0 \times 10^{2} \leq f_{c} \leq 2.5 \times 10^{3}$ $\mathrm{Hz}$ and $2.0 \leq Q \leq 33.3$, and take the minimal match $M M=0.98$. The total number of templates $\mathcal{N}$ amounts to 628 . To analyze a data chunk of length $52 \mathrm{sec}$, it takes about 130 seconds with the 628 filters using a single CPU (Intel Pentium-IV 2.5 GHz). The TAMA300 data have analyzed with sixteen-node PC clusters at NAOJ and OCU. The time required for a full-scale search using 1000 hours data is about one week.

In this work, we use the template bank generated so that the minimal match is assured when the noise power spectrum is white i.e. $S_{n}(|f|)=$ const. in the calculation of correlations of two waveforms by equation (4). It must be noted that since the noise power spectrum of a real detector is not stationary, the metric of the parameter space and appropriate template spacings also change in time. Nevertheless we apply the same template bank to all the data chunks used here. This is supported by the fact that ringdown waveforms have Fourier spectra of rather narrow band centered at $f_{c}$ (see FIG. 1), which is especially true for large $Q$ ringdowns, and the match given by equation (4) is expected to be less sensitive to whole shape of the noise power spectrum $S_{n}(|f|)$. This has been confirmed in [21] with model calculations using the TAMA300 fitted noise curve as described above (similar discussion is also found in [19]). Thus the question is if the template bank based on white noise power spectrum can still be valid or effective enough when applied to real observational data that contain time variation of detector's noise. 


\section{MATCHED FILTERING SIMULATION USING TAMA300 DATA}

\section{A. Monte-Carlo Simulation of Galactic Ringdown Events}

In this section, we describe our simulations in detail. Randomly generated ringdown signals are injected into TAMA data, and filtered with the template bank. For gravitational wave sources, we consider Galactic black holes, since TAMA300 has enough sensitivity to detect them thus they are suitable target for our search. The spatial distribution of sources in the Galaxy is assumed as a form in the Galacto-centric cylindrical coordinates $(R, z, \varphi)$,

$$
\mathrm{d} N \propto \exp \left(-2 R^{2} / R_{0}^{2}\right) \exp \left(-|z| / h_{z}\right) R \mathrm{~d} R \mathrm{~d} z \mathrm{~d} \varphi
$$

where $R_{0}=4.8 \mathrm{kpc}$ and $h_{z}=1 \mathrm{kpc}$ [24]. The spin axes of black holes are assumed to be randomly directed. It is rather difficult to estimate the absolute amplitudes of ringdown waves. Flanagan and Hughes have given an estimate on a fractional energy of the black hole masses radiated as gravitational waves to be about 3\% [25], and we use this value for determination of the signal amplitudes. The injection point $t_{0}$ is randomly selected in time of the TAMA300 observation periods. Once $t_{0}$ is given, the sky position of the source in the horizontal coordinates $(\theta, \phi)$ is obtained from the source position in the $(R, z, \varphi)$ coordinates, and then the antenna pattern function in the direction $(\theta, \phi)$ is calculated. The amplitude of the signal at the detector is determined from the distance to the source, the antenna pattern function, and the angular distribution of the gravitational radiation determined by the spin-weighted spheroidal harmonics ${ }_{-2} S_{22}(\cos i, a)$, where $i$ is the inclination angle of the black hole axis seen from the observer. The ringdown parameters $\left(f_{c}, Q\right)$ of the simulated signals are uniformly chosen in ranges $f_{c}=100 \sim 2500 \mathrm{~Hz}$ and $Q=2 \sim 33.3$ (FIG. 2 3). The range of $f_{c}$ is determined considering the observational band of TAMA300 (see FIG. 1). The range of $Q$ corresponds to the Kerr parameter $a=0 \sim 0.998$. The distribution of black hole masses (mass function) of the simulated ringdown signals results in $\propto M^{-2}$, since $f_{c}$ is uniformly selected and $M$ is inversely proportional to $f_{c}$.

A simulated signal is superimposed on a data chunk of $52 \mathrm{sec}$ long at a randomly selected injection point $t_{0}$ with an initial phase (FIG. 4), and the data chunk is filtered with each template of the bank, and also with the waveform of the simulated signal itself. The inputs for each event are the signal parameters $\left(f_{c}, Q\right)$, and the outputs are the matches for all of the filters used. Here the match for each template is given by equation (4) using the value of the filter output $\rho$ at the local maximum found just around the injection point $t_{0}$. The SNR of an event is defined as the greatest in the matches, and the signal parameters are determined as those of the bestmatched template. The TAMA300 data used here is ob-

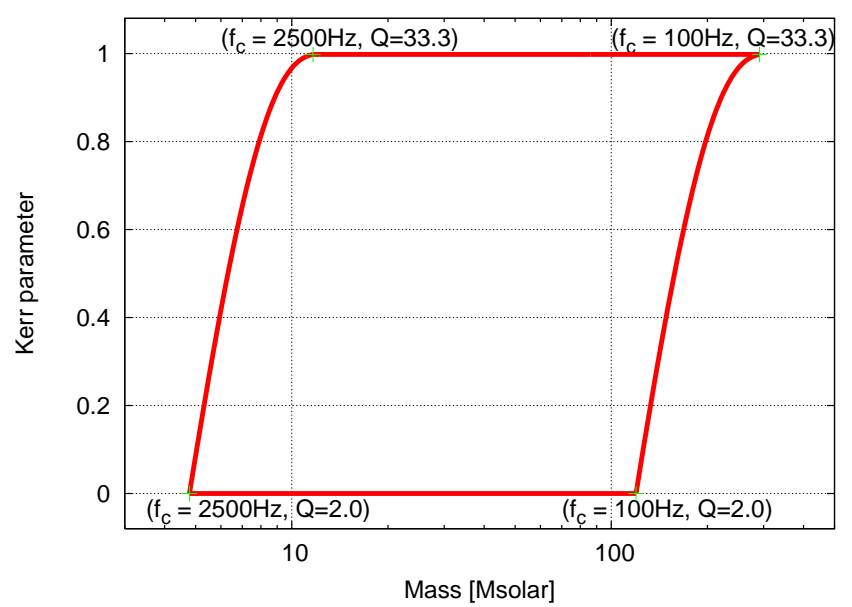

FIG. 2: Parameter range. We generate ringdown signals with parameters $f_{c}=100 \sim 2500 \mathrm{~Hz}, Q=2 \sim 33.3$ uniformly chosen in $\left(f_{c}, Q\right)$ plane.

tained from 2001/Sep/17 to 2001/Sep/20 in TAMA Data Taking 6 (DT6), and from 2003/Feb/13 to 2003/Feb/17 in DT8. For each data chunk, 200 simulated signals are examined individually, and these procedures are carried out for all the data chunks. The noise power spectrum of the detector outputs $S_{n}(|f|)$ required in the calculation of the match is evaluated for each data chunk with the moving average method using the preceding data chunks. Note that we use the fixed template bank for all the data chunks, as described in section $1 \mathrm{~A}$ and the amplitude of each template is normalized so as to be $\langle h \mid h\rangle=1$ in equation (4).

This simulation method has some advantages in evaluating the detection efficiency or the detection probability for the Galactic events compared to the case with analytical calculations using a Galactic modeling and a typical detector sensitivity. Since we use the real observational data, we can include the time variation of detector's noise fluctuation and the sensitivity. Moreover, we also follow the antenna direction in the Galaxy at all times, thus we can properly take into account of the operational conditions of the detector to any Galactic events which would have occurred during the observation periods.

\section{RESULTS}

\section{A. Detection Efficiency}

By examining the SNRs of simulated ringdown events, we evaluate the detection efficiency $\epsilon$ for a given SNR threshold. Since we assume the sources are distributed in the Galaxy as equation (5), this can be interpreted as the detection probability for the Galactic ringdown events. For example, if we detect gravitational wave events $N_{\text {obs }}$ in observation time $T$ and assume all of them 


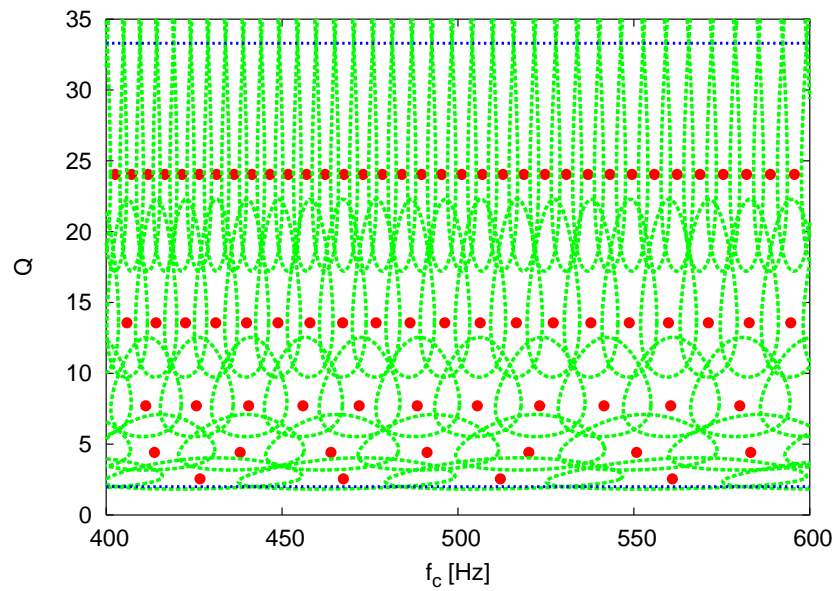

FIG. 3: A part of the template bank generated under the condition of minimal match $M M=0.98$. The templates are placed along fixed $Q$ lines $(Q=2.6,4.4,7.7,13.6,24.0)$ by using the tiling method proposed in [20]. The oval for each of the templates shows the tiled region in which mismatches between the template and any waveforms do not exceed $2 \%$ in the case of white noise. There is no uncovered region in the parameter ranges $f_{c}=100 \sim 2500 \mathrm{~Hz}$ and $Q=2 \sim 33.3$. The ratio of the total area of the tiles to that of the parameter space covered is 1.57 .

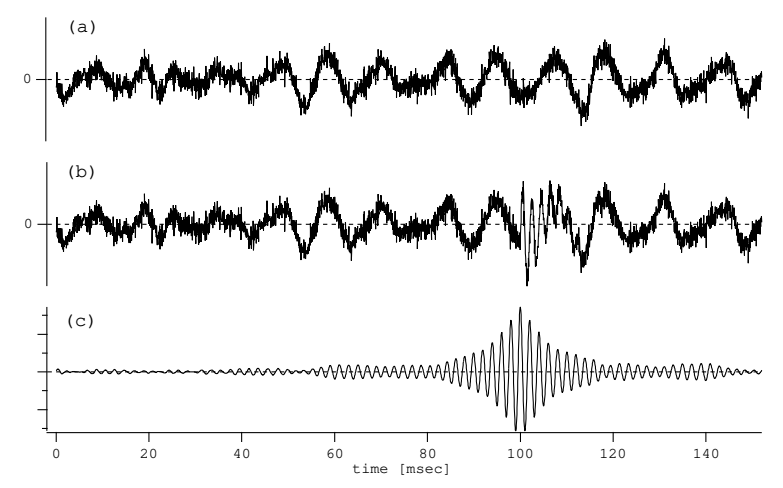

FIG. 4: Schematic view of software signal injection into real data (in time domain): (a) strain data $n(t)$ from the interferometer, (b) the strain data $n(t)$ and a simulated signal $s(t)$ with waveform parameters $f_{c}=500[\mathrm{~Hz}], Q=20$ injected at $t_{0}=100[\mathrm{msec}],(\mathrm{c})$ matched filter output $\langle n+s \mid s\rangle$.

are of Galactic origin, the Galactic ringdown event rate is estimated as $N_{\text {obs }} /(\epsilon T)$. The detection probabilities in DT6 and DT8 as functions of the ringdown frequency $f_{c}$ are displayed in FIG. 5 for different SNR thresholds, SNR $\geq 6, \geq 10$, and $\geq 30$. All the conditions which affect to the detection probabilities such as the template spacings, noise fluctuations and the position of the observer are properly taken into account. The detection probabilities are significantly improved in DT8, in particular for higher $f_{c}$ events. This is attributed to power-recycling

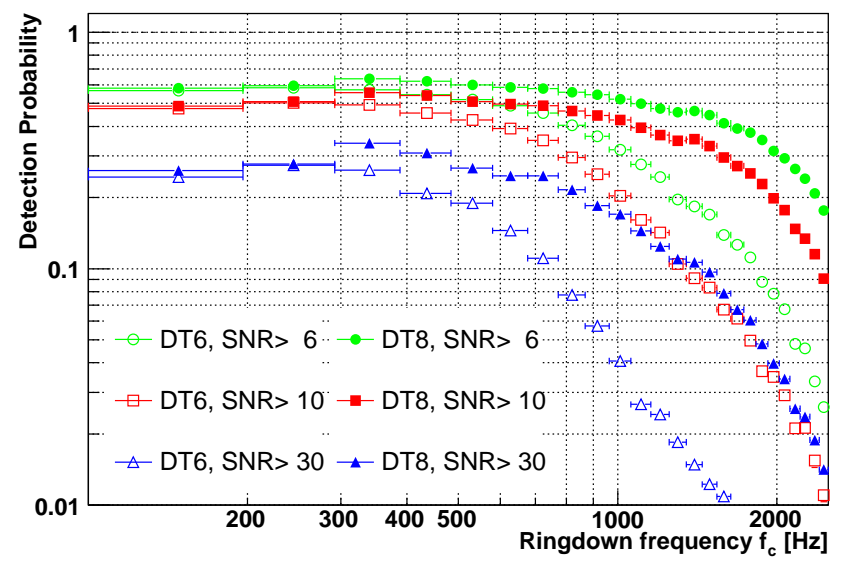

FIG. 5: Detection probability for Galactic events, for DT6 (open symbols) and DT8 (closed symbols). Circle: $\mathrm{SNR} \geq 6$, Square: $\mathrm{SNR} \geq 10$, Triangle: $\mathrm{SNR} \geq 30$

implemented after DT6 to increase the input laser power in the cavities of the interferometer and reduce the shot noise which is dominant in higher frequencies. We can detect about $\epsilon=50 \%$ of the Galactic ringdown events of frequencies $f_{c} \leq 600 \mathrm{~Hz}$ (corresponding black hole mass $\geq 20 \mathrm{M}_{\odot}$ ) under the criterion $\mathrm{SNR} \geq 10$.

We note that these results depend on the evaluation of absolute amplitudes of the ringdown waves at the sources. We assume that the fractional mass loss of a black hole by radiating gravitational waves is $3 \%$ according to Flanagan and Hughes, and all of the energies are carried by the waves of the least damped mode of the QNMs.

\section{B. Matched Filtering and Parameter Determination}

Next we consider matches between waveforms and templates, and accuracies in determination of the waveform parameters. It is expected that these are primarily limited by the template spacings. We recall here the definition of the match between a template $h$ and a signal $s$ injected in detector output $n$ :

$$
\rho=\langle o \mid h\rangle \propto \int d f \frac{\tilde{o}(f) \tilde{h}^{*}(f)}{S_{n}(|f|)}
$$

where $\tilde{o}(f)=\tilde{n}(f)+\tilde{s}(f)$. For each of our simulated signals, we can define three points in the parameter space, those are (1) a priori template with the same parameter values of the simulated signal, $\mathbb{S}:\left(f_{c}^{S}, Q^{S} ; \rho^{S}=\right.$ $\langle o \mid \mathbb{S}\rangle),(2)$ the nearest to $\mathbb{S}$ in the template bank, $\mathbb{N}$ : $\left(f_{c}^{N}, Q^{N} ; \rho^{N}=\langle o \mid \mathbb{N}\rangle\right)$, and (3) the best-matched template, $\mathbb{M}:\left(f_{c}^{M}, Q^{M} ; \rho^{M}=\langle o \mid \mathbb{M}\rangle\right)$, which is the maximum of the match in the bank and gives the SNR for the signal. The template we can single out from the bank 
is $\mathbb{M}$, not the nearest $\mathbb{N}$, but the implicit assumption in matched filtering is that the two templates $\mathbb{N}, \mathbb{M}$ are identical and the parameters $f_{c}^{N}=f_{c}^{M}, Q^{N}=Q^{M}$ are the best estimates for $\mathbb{S}$. Note that the metric of the parameter space which defines the distances between the two templates depends on the noise power spectrum $S_{n}(|f|)$. In the template construction we employ in this work, the metric of the parameter space is given on the assumption of white noise, $S_{n}(|f|)=$ constant. The template spacing is carried out in order that for any signals of waveform parameters within the search range, we can find at least one template such that the match is greater than the minimal match $M M$, i.e. $\rho^{M} / \rho^{S} \geq M M$. Thus if the noise power spectrum is colored, the definition of the distance is changed and hence the maximum of the match $\rho^{M} / \rho^{S}$ could be smaller than $M M$. Nakano et al. has investigated the matches between the templates and signals with randomly selected waveform parameters, $\langle s \mid \mathbb{N}\rangle$, by direct integration of equation (6) using an analytical fit of the TAMA300 typical noise curve (no lines, no fine structures), and showed that the fraction of signals which give $\rho^{M} / \rho^{S}<M M$ is only $0.88 \%$ (21], see also section IA). Therefore we use the fixed template bank in the matched filtering analysis using TAMA300 data, for all the data chunks at different detector conditions.

However, the noise power spectrum of a real detector $S_{n}(|f|)$ has many lines and fine structures, and changes its shape with time. Moreover, the detector noises are not stationary and signals would be contaminated by burstlike noises. Thus it is not clear whether the criterion $\rho^{M} / \rho^{S} \geq M M$ is still fulfilled in a practical search using the fixed template bank. An alternative method is use of adaptive template banks, determined from the actual noise power spectrum and metric of the parameter space, as performed in neutron star binary search [26]. Our approach makes the data analysis codes simpler, but the effect of noise fluctuations must be considered carefully. Furthermore, when the noise power spectrum $S_{n}(|f|)$ is deformed and the signal $\tilde{s}(f)$ in equation (6) falls in a noisy frequency region, the match is degraded and the template $\mathbb{M}$ selected from the bank does not necessarily coincide with the nearest template $\mathbb{N}$ (see FIG. [6). In this case, the errors in parameter determination could be larger than those limited by the template spacings.

\section{Finding Best-Matched Template}

To investigate these effects, we examine the ratios $\rho^{N} / \rho^{S}=\langle o \mid \mathbb{N}\rangle /\langle o \mid \mathbb{S}\rangle$ and $\rho^{M} / \rho^{S}=\langle o \mid \mathbb{M}\rangle /\langle o \mid \mathbb{S}\rangle$, those are measures of the utility of the template bank. If the noise power spectrum is white $\left(S_{n}(|f|)=\right.$ const.) and there are no non-stationary noises, the maximum value of $\rho^{N} / \rho^{S}$ is unity and its distribution has a cut-off at the minimal match $M M=0.98$ defined in the template construction. The distribution of $\rho^{N} / \rho^{S}$ for the TAMA300 data-injected signals are shown in FIG. 7 (a). For a reference the distribution of $\langle s \mid \mathbb{N}\rangle$ calculated by using an analytical fit of the TAMA300 typical noise curve 21] is also displayed. The tail of the distribution of $\rho^{N} / \rho^{S}$ in the lower side looks in agreement to that expected from the template design, $\langle s \mid \mathbb{N}\rangle$. The fraction of events with $\rho^{N} / \rho^{S}<M M$ is $3.3 \%$, which is larger than $0.88 \%$ for $\langle s \mid \mathbb{N}\rangle$ because of the noise effects. We note that about $10 \%$ of simulated signals have the fractional match $\rho^{N} / \rho^{S}$ greater than 1 (many of them are observed in lower $Q$ signals), and the mean of $\rho^{N} / \rho^{S}$ is 0.994 , slightly larger than that of $\langle s \mid \mathbb{N}\rangle, 0.993$. This is related to contaminations of the signals by the noise $n$, and the estimated noise power spectrum $S_{n}(|f|)$ used in matched filtering. If the true noise power spectrum at the time when the data $n$ was obtained differs from the estimated spectrum $S_{n}(|f|), \rho^{N} / \rho^{S}>1$ is possible since each template is normalized as $\langle h \mid h\rangle=1$ by using the estimated spectrum $S_{n}(|f|)$. Therefore in a realistic gravitational wave search there are inevitable ambiguities in evaluation of matches (or SNRs) and their losses.

In FIG. 7 (b), the distribution of the ratio $\rho^{M} / \rho^{S}$ is shown. The mean of $\rho^{M} / \rho^{S}$ is 0.998 , and the fractions of events of $\rho^{M} / \rho^{S}<M M$ and $\rho^{M} / \rho^{S}>1$ are $0.0012 \%$ and $24 \%$, respectively. This indicates that our template bank fulfills the requirement of the minimal match $>M M$ even in practical search, and also it is difficult to estimate the "true" value of the match, $\langle s \mid \mathbb{S}\rangle$ or $\langle s \mid \mathbb{N}\rangle$, within accuracies of a few $\%$. The occurrence probability of $\mathbb{N} \neq \mathbb{M}$ is about $30 \%$ (see FIG. 8). This causes increases of waveform parameter determination errors.

\section{Waveform Parameter Errors}

The accuracies in determination of the waveform parameters $\left(f_{c}, Q\right)$ are evaluated by comparing those of the simulated signal $\mathbb{S}$ and of the best-matched template $\mathbb{M}$. The root mean squares (RMSs) of the errors $\left(\Delta f_{c} / f_{c}\right)_{\mathrm{RMS}} \equiv\left(\left(f_{c}^{M}-f_{c}^{S}\right) / f_{c}^{S}\right)_{\mathrm{RMS}}$ and $(\Delta Q / Q)_{\mathrm{RMS}} \equiv\left(\left(Q^{M}-Q^{S}\right) / Q^{S}\right)_{\mathrm{RMS}}$ are calculated as $1.3 \%$ and $22 \%$, respectively. These are comparable to RMSs of $\left(\Delta f_{c} / f_{c}\right)_{\mathrm{RMS}}=1.22 \%$ and $(\Delta Q / Q)_{\mathrm{RMS}}=$ $16.3 \%$ estimated from the template spacings, but somewhat broadened. This is because for not all the events the template $\mathbb{M}$ coincides with the nearest $\mathbb{N}$, as seen in FIG 8 In practice, $\left(\Delta f_{c} / f_{c}\right)_{\mathrm{RMS}}$ and $(\Delta Q / Q)_{\mathrm{RMS}}$ depend on $Q$, as the template distances are (see FIG. 3). The values of $\left(\Delta f_{c} / f_{c}\right)_{\mathrm{RMS}}$ and $(\Delta Q / Q)_{\mathrm{RMS}}$ are listed in TABLE【

\section{DISCUSSION}

\section{A. Black Hole Spectroscopy}

The distances between the ringdown filters determined from the requirement to the minimal match $M M$ limit the waveform parameter resolutions. The resolutions for the ringdown parameters in matched filtering analysis 
SNR Contour: Event 97, fc $=1197.6, Q=9.2$, SNR $=16.0$

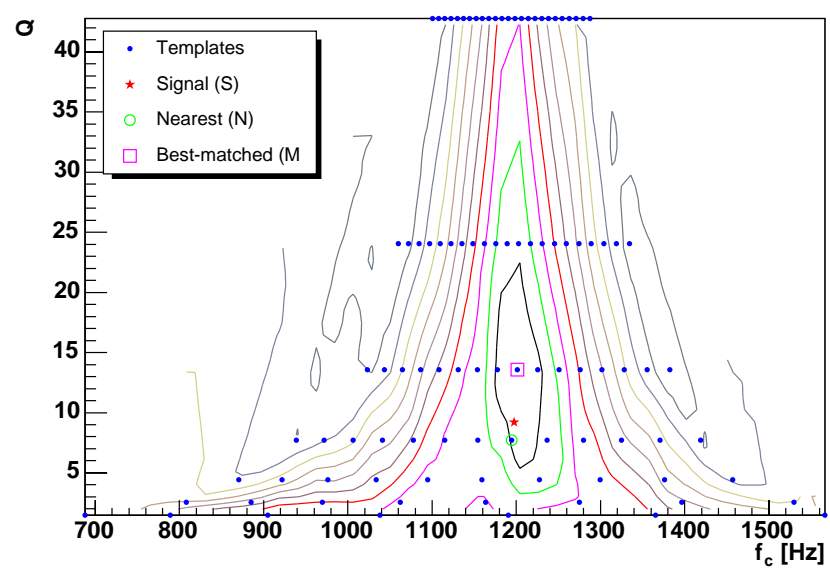

FIG. 6: An example of contour map of SNRs in the parameter space. The star is the simulated signal $\mathbb{S}$, which falls at $\left(f_{c}=1197.6 \mathrm{~Hz}, Q=9.6\right)$, and the solid points represent the templates around $\mathbb{S}$. The circle indicates the nearest template $\mathbb{N}$, and the square shows the best-matched template $\mathbb{M}$ which gives the maximum of SNR. In most of events the two templates $\mathbb{N}$ and $\mathbb{M}$ coincide, but differ in this sample. This is due to an additional "curvature" of the parameter space introduced by the noise power spectrum $S_{n}(|f|)$, and contamination of the signal by non-stationary noises.
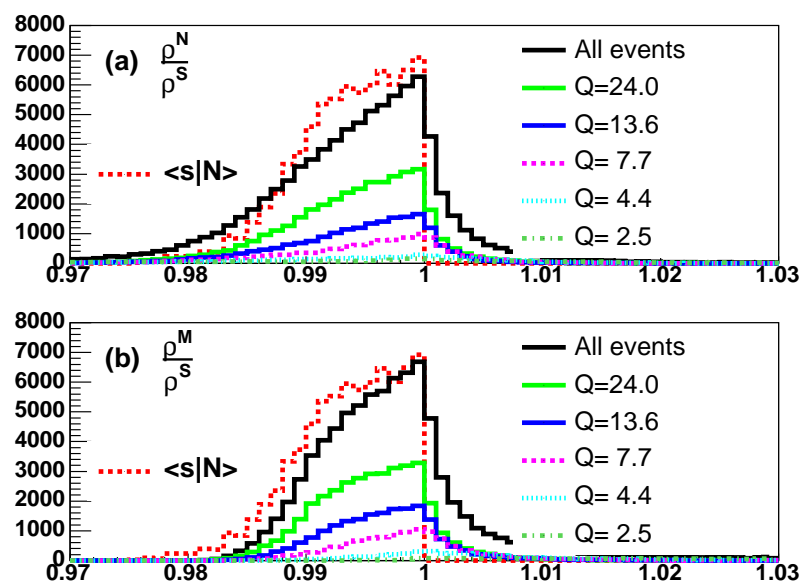

FIG. 7: Distributions of ratios of the SNRs, $\rho^{N} / \rho^{S}=$ $\langle o \mid \mathbb{N}\rangle /\langle o \mid \mathbb{S}\rangle$ and $\rho^{M} / \rho^{S}=\langle o \mid \mathbb{M}\rangle /\langle o \mid \mathbb{S}\rangle$ (see text for definitions). The broken line is distribution of $\langle s \mid \mathbb{N}\rangle$, where $s$ is a randomly generated ringdown waveform, obtained by direct integration of equation (6) using a typical TAMA300 noise curve [21].

with the template bank of $M M=0.98$ using TAMA300 data are $\left(\Delta f_{c} / f_{c}\right)_{\mathrm{RMS}}=1.3 \%$ and $(\Delta Q / Q)_{\mathrm{RMS}}=22 \%$ as described in the previous section. The question here is how accurately we can determine the source parameters, the mass $M$ and the Kerr parameter $a$, from the results of matched filtering analysis. By using the Echeverria's

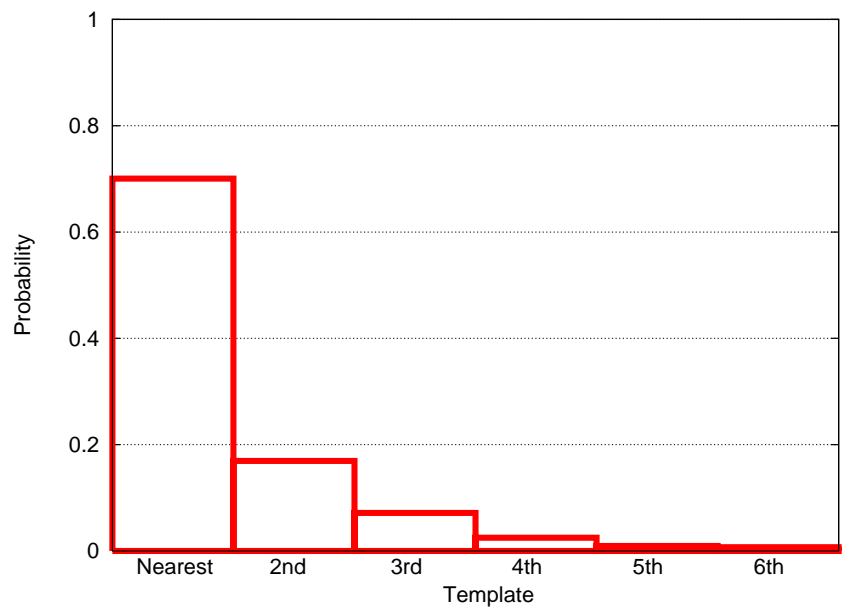

FIG. 8: Distribution of the templates which give the maximum of SNRs in the bank.

expressions (2) and (3), we found $(\Delta M / M)_{\mathrm{RMS}}=10 \sim$ $20 \%$ for low $Q$ signals, and a few $\%$ for higher $Q$ ringdowns. The error in the Kerr parameter determination is about $(\Delta a / a)_{\mathrm{RMS}}=40 \sim 60 \%$. The calculated values of $(\Delta M / M)_{\mathrm{RMS}}$ and $(\Delta a / a)_{\mathrm{RMS}}$ are listed in TABLE $\square$

Obviously these results depend on the template design, i.e. template spacings. It is expected that the parameter resolution can be improved as template distances become closer, and saturates when the mismatches between signals and neighboring templates approach to intrinsic ambiguities limited by the noise fluctuations. In case of Gaussian noise, the intrinsic parameter estimation errors can be evaluated by using the Fisher information matrix 27, 28]. For example, for ringdown signals of $\left(f_{c}=580 \mathrm{~Hz}, Q=24\right)$, these are calculated as functions of SNRs, as $\left(\Delta f_{c} / f_{c}\right)_{\mathrm{RMS}}=0.30 \%$ and $(\Delta Q / Q)_{\mathrm{RMS}}=14 \%$ at $\mathrm{SNR}=10$ and inversely proportional to SNRs (see Appendix B. in [21]). To confirm this, we performed matched filtering simulations for fixed parameter ringdown signals at $f_{c}=580 \mathrm{~Hz}, Q=24.0$ $\left(M=50 M_{\odot}, a=0.996\right)$, with very fine-meshed templates uniformly distributed around the signal. We found $\left(\Delta f_{c} / f_{c}\right)_{\mathrm{RMS}}=0.21 \%$ and $(\Delta Q / Q)_{\mathrm{RMS}}=9.3 \%$ for signals of SNR $=10 \sim 20$ (FIG 9). The parameter estimation errors are in agreement with the predictions of the Fisher information matrix, and small excursions from them are considered due to non-Gaussian component of the TAMA300 data. With these results, the black hole parameter estimation errors are evaluated as $(\Delta M / M)_{\mathrm{RMS}} \sim 0.9 \%,(\Delta a / a)_{\mathrm{RMS}} \sim 24 \%$ for $\mathrm{SNR} \sim 10$, and $(\Delta M / M)_{\mathrm{RMS}} \sim 0.3 \%,(\Delta a / a)_{\mathrm{RMS}} \sim 7 \%$ for SNR $\sim 50$. No further improvements are expected even in a case of more finer mesh, since these limits are from the intrinsic ambiguities in matches due to the noise fluctuations. (Note that since the accuracies in the conversions from $\left(f_{c}, Q\right)$ to $(M, a)$ are only $\sim 5 \%$, we need more accurate formulae.) 


\begin{tabular}{|c||cc|cc|}
\hline$Q^{M}$ & $\left(\Delta f_{c} / f_{c}\right)_{\mathrm{RMS}}(\Delta Q / Q)_{\mathrm{RMS}}$ & $(\Delta M / M)_{\mathrm{RMS}}(\Delta a / a)_{\mathrm{RMS}}$ \\
\hline All & $1.3(1.2)$ & $22(16)$ & & \\
2.55 & $8.1(2.6)$ & $22(16)$ & $22(12)$ & $64(35)$ \\
4.41 & $4.0(1.6)$ & $24(16)$ & $13(6.6)$ & $41(35)$ \\
7.70 & $1.6(1.0)$ & $21(16)$ & $6.8(3.9)$ & $39(36)$ \\
13.6 & $0.77(0.58)$ & $19(16)$ & $3.1(2.4)$ & $40(36)$ \\
24.0 & $0.39(0.33)$ & $19(17)$ & $1.9(1.6)$ & $41(37)$ \\
\hline
\end{tabular}

TABLE I: RMS errors in estimations of ringdown - black hole parameters (in \%) by using the template bank of $M M=$ 0.98. The quantities are defined as $\Delta X / X \equiv\left(X^{M}-\right.$ $\left.X^{S}\right) / X^{S},\left(X=f_{c}, Q, M, a\right)$. For comparison, analytic estimations from the template distances, which should be obtained in the absence of noise fluctuations, are also shown (parenthesized). The parameter conversions $\left(f_{c}, Q\right) \leftrightarrow(M, a)$ are based on the Echeverria's expressions in equations (2) and (3).

When we find event candidates of ringdown signals, we can determine the black hole parameters within the accuracies described above by setting a fine-meshed template bank locally at each of the event candidates. There is no need to cover whole parameter space with the template bank of such closer distances, the hierarchical search strategy copes with computational costs [29]. The resolutions for the black hole parameters obtained here are remarkably good, compared to those estimated dynamically for known black hole candidates in X-ray binaries or radio sources, typically $10 \sim 100 \%$ [30]. Some X-ray binaries as GRS1915+105 eject superluminal jet, and rotating black holes are considered as the central engine [31]. Precise determination of the Kerr parameter $a$ of black holes by observing gravitational waves will give strong constrains on jet formation mechanisms [32, 33, 34]. Thus gravitational wave observations provide not only a probe for black hole detections, but also a promising clue to establish black hole "spectroscopy".

\section{CONCLUSIONS}

Black hole ringdowns are promising source of gravitational radiation, and the matched filtering technique is suitable to extract ringdown signals from outputs of gravitational wave detectors. In this paper, we studied template constructions, detection efficiencies and waveform parameter estimations in matched filter searches using TAMA300 data. In template construction, we employed a simple and efficient tiling method proposed by Nakano et al. 20, 21]. The template bank constructed with this method assures the minimal match criterion for any ringdown waveforms in the cases of a flat noise power spectrum $S_{n}(|f|)=$ const. and a colored spectrum fitted to TAMA300 typical noise curve [20, 21]. The errors in waveform parameter estimation are determined by the template separations hence the choice of $M M$. In
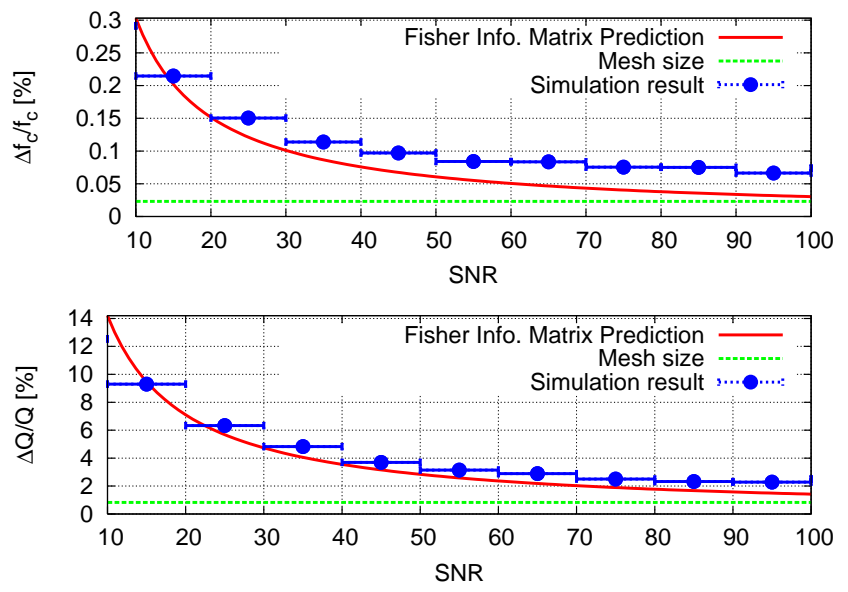

FIG. 9: Waveform parameter resolutions in the case of a finely meshed template bank (closed circle). The simulation is carried out for a fixed waveform with parameters $f_{c}=580 \mathrm{~Hz}$ and $Q=24$. The templates are uniformly placed around the signal with separations of $\Delta f_{c}=0.13 \mathrm{~Hz}\left(\Delta f_{c} / f_{c}=0.023 \%\right)$ and $\Delta Q=0.2(\Delta Q / Q=0.83 \%)$ (broken line). The predictions from the Fisher information matrix, which are expected in cases of Gaussian noises are also shown (solid line).

gravitational wave searches using real observational data, however, it is not clear whether the minimal match criterion is fulfilled since non-stationary noises contaminate signals and the time-varying noise power spectra change matches or distances between signals and the templates. In a such case, the nearest template and the best-matched template for a signal do not necessarily coincide and parameter estimation errors increases.

To investigate these effects, we performed matched filtering analysis for artificial ringdown signals injected into TAMA300 data with Monte-Carlo technique. We consider black holes with masses $10 \sim 200 M_{\odot}$ distributed in the Galaxy as "realistic" search targets. With TAMA DT8 sensitivity and using our template bank, the detection probability is evaluated as $\sim 50 \%$ for Galactic ringdown events of $f_{c}<600 \mathrm{~Hz}$, which correspond to black holes with masses $\geq 20 M_{\odot}$, assuming the fractional black holes mass $\sim 3 \%$ radiated as gravitational waves. We confirmed that the loss of SNR is less than $2 \%$ in most cases for ringdown signals embedded in TAMA300 data, therefore we can use the template bank in gravitational wave searches using real observational data. We also showed that it is difficult to evaluate matches $\rho$ or SNRs within an accuracy of a few $\%$ because of the signal contaminations and the difficulties in estimation of "true" noise power spectrum at given observation time. The RMS errors in the waveform parameter estimations are examined by comparing the simulated signals and the templates singled out from the bank. We obtained $\left(\Delta f_{c} / f_{c}\right)_{\mathrm{RMS}}=1.3 \%$ and $(\Delta Q / Q)_{\mathrm{RMS}}=22 \%$, which are comparable with the expectations from the template spacings, $\Delta f_{c} / f_{c}=1.22 \%$ and $\Delta Q / Q=16.3 \%$, respec- 
tively. The informations obtained in this work as the detection probability and the parameter estimation errors will be used in statistical and astrophysical interpretation of results from the TAMA300 full-scale event search, which is under progress. The key in the event search is to develop efficient vetoing techniques to distinguish real signals and spurious triggers due to impulsive noises [35].

Our interest extends to how accurately we can determine the waveform parameters hence the black hole masses and the angular momenta by using an extremely fine-meshed template bank. For ringdown signals of $f_{c}=580 \mathrm{~Hz}, Q=24.0$, the ultimate resolutions are evaluated as $\left(\Delta f_{c} / f_{c}\right)_{\mathrm{RMS}}=0.21 \%$ and $(\Delta Q / Q)_{\mathrm{RMS}}=9.3 \%$ for signals of SNR $=10 \sim 20$, which are consistent with the predictions of the Fisher information matrix. The errors in black hole parameter estimations are calculated as $(\Delta M / M)_{\mathrm{RMS}} \sim 0.9 \%,(\Delta a / a)_{\mathrm{RMS}} \sim 24 \%$ for $\mathrm{SNR} \sim 10$, and $(\Delta M / M)_{\mathrm{RMS}} \sim 0.3 \%,(\Delta a / a)_{\mathrm{RMS}} \sim 7 \%$ for SNR $\sim 50$. In a practical search, we will employ the hierarchical search strategy, performing the first event search with an "ordinary" template bank, of minimal match $M M=0.98$ for example, and the second search for event candidates with a locally fine-meshed template bank. The remarkably good resolutions for $(M, a)$ are heartening to understand gravitational physics and astrophysics of black holes.

\section{Acknowledgments}

The authors wish to acknowledge to all the collaborators in TAMA project. YT expresses thanks to T. Akutsu, K. Arai, M. Fujimoto, K. Hayama, S. Sato, K. Somiya, and R. Takahashi for helpful comments and encouragements. HN would like to thank H. Ishihara and K. Nakao for useful discussions. This research is partially supported by a Grant-in-Aid for Creative Basic Research (09NP0801), for Scientific Research on Priority Areas (415), and for Scientific Research No. 16540251 of the Ministry of Education, Culture, Sports, Science and Technology. HN is supported by the Japan Society for the Promotion of Science for Young Scientists (No. $5919)$.

\section{APPENDIX A: PRECEDING WAVEFORMS}

So far we have assumed that there are no preceding waves before the ringdown signal arrives. If the ringdown is initiated by a binary coalescence, however, we would receive gravitational waves of characteristic waveforms successively, as the chirp signal at first, the waves emitted in the merger phase, followed by the ringdown signal in the last stage. To investigate effects of the preceding waves, we performed a test of matched filtering for a such composite waveform injected in TAMA300 data. The aim of this is not to generate realistic gravitational waveform from a binary coalescence, nor to use composite

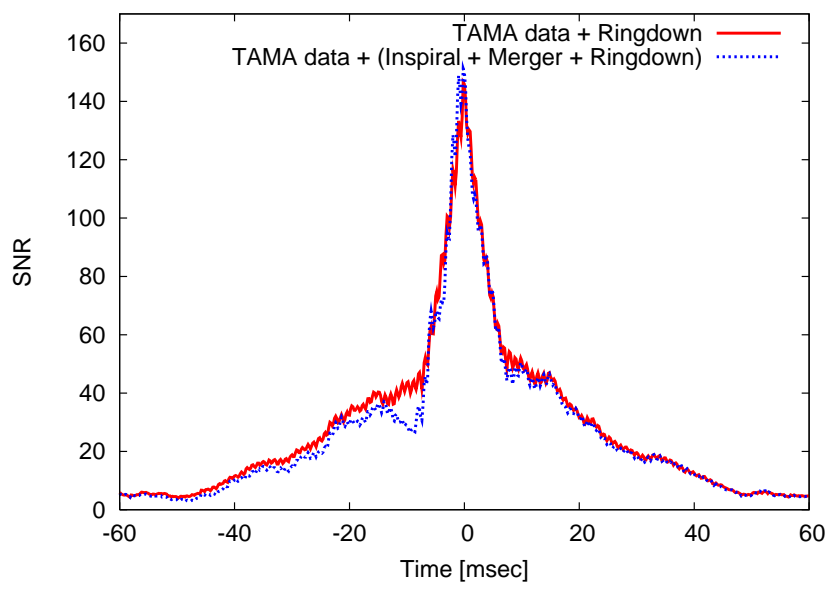

FIG. 10: An example of ringdown filter outputs in time series for a ringdown signal (solid) and for a composite waveform (broken) injected in a TAMA300 data chunk.

waveforms as templates, but to examine whether we can extract ringdown signals alone from the composite waveform embedded in detector outputs by using our ringdown filters. Here we considered binary coalescence of masses $25 M_{\odot}-25 M_{\odot}$ to form a black hole of $50 M_{\odot}$ with a Kerr parameter $a=0.98\left(f_{c}=520 \mathrm{~Hz}, Q=12\right)$. In this case, the cut-off frequency of the inspiral phase is below $100 \mathrm{~Hz}$, therefore the contribution of the chirp signal is small. Although the waveforms in the merger phase are quite uncertain, we presume sine-Gaussian waveform, as commonly used in the burst search studies, of the central frequency $\sim 300 \mathrm{~Hz}$ with amplitude of twice of the ringdown signal and a phase to be connected analytically with the following ringdown. We found that (i) the maximum of SNR is found at the template nearest to the simulated signal, (ii) the peak of the filter output in time series is found at which ringdown begins, and (iii) the value of SNR is consistent within $1 \%$ compared to that in the case of no preceding waveforms injected (FIG. 10). This is just a preliminary inspection on effects of the preceding waveforms in a ringdown search, and we need to know more physics of the merger phase for quantitative examinations.
[1] P. Astone et al., Phys. Rev. D 68, 022001 (2003).

[2] R. Takahashi, Class. Quantum. Grav. 21, S403 (2004).
[3] B. Abbot et al., Nucl. Instrum. Meth. A 517, 154 (2004).

[4] B. Willke et al., Class. Quantum. Grav. 21, S417 (2004). 
[5] T. Pradier et al., Phys. Rev. D 63, 042002 (2001).

[6] J. Sylvestre, Phys. Rev. D 66, 102004 (2002).

[7] T. Regge and J. Wheeler, Phys. Rev. 108, 1063 (1957).

[8] F. Zerilli, Phys. Rev. D 2, 2141 (1970).

[9] S. Teukolsky, Phys. Rev. Lett. 29, 1114 (1972).

[10] S. Teukolsky, Astrophys. J. 185, 635 (1973).

[11] S. Detweiler, Astrophys. J. 239, 292 (1980).

[12] S. Chandrasekhar and S. Detweiler, Proc. R. Soc. Lond. A344, 441 (1975).

[13] V. Ferrari and B. Mashhoon, Phys. Rev. Lett. 52, 1361 (1984).

[14] B. Schutz and C. Will, Astrophys. J. Lett. 291, L33 (1985).

[15] K. Kokkotas and B. Schmidt, Living Rev. Relativity [Online article] 2, 2 (1999).

[16] E. Leaver, Proc. R. Soc. Lond. A402, 285 (1985).

[17] F. Echeverria, Phys. Rev. D 40, 3194 (1989).

[18] J. Creighton, Phys. Rev. D 60, 022001 (1999).

[19] N. Arnaud et al., Phys. Rev. D 67, 102003 (2003).

[20] H. Nakano et al., Phys. Rev. D 68, 102003 (2003).
[21] H. Nakano et al., Prog. Theor. Phys. 111, 781 (2004).

[22] B. Owen, Phys. Rev. D 53, 6749 (1996).

[23] D. Tatsumi and Y. Tsunesada, Class. Quantum Grav. 21, S451 (2004).

[24] S. Curran and D. Lorimer, Mon. Not. R. Astron. Soc. 276, 347 (1995).

[25] E. Flanagan and S. Hughes, Phys. Rev. D 57, 4535 (1998).

[26] H. Takahashi et al., Phys. Rev. D 70, 042003 (2004).

[27] L. S. Finn, Phys. Rev. D 46, 5236 (1992).

[28] C. Cutler and E. Flanagan, Phys. Rev. D 49, 2658 (1994).

[29] S. Mohanty, Phys. Rev. D 57, 630 (1997).

[30] J. Ziólkowski, pp. astro-ph/0307307 (2003).

[31] J. Greiner et al., Nature 414, 522 (2001).

[32] S. Koide et al., Astrophys. J. 536, 668 (1999).

[33] J. D. Villiers et al., pp. astro-ph/0407092 (2004).

[34] A. Tomimatsu et al., Phys. Rev. D 64, 123003 (2001).

[35] Y. Tsunesada et al., Class. Quantum Grav. 21, S703 (2004). 